JOURNAL CLUB

\title{
Hospitalisation rates for pneumonia following the introduction of a pneumococcal vaccination
}

In response to concerns regarding increases in non-vaccine serotype-related pneumococcal disease, a 7-valent pneumococcal conjugate vaccine (PCV7) was introduced to the US child immunisation schedule in 2000. This study evaluated the impact of this vaccine on hospitalisation rates for pneumonia in the decade following its introduction.

Interrogation of the Nationwide Inpatient Sample database allowed comparison of pneumonia-related hospitalisation rates in the 3-year period prior to the introduction of the PCV7 with rates between 2007 and 2009. Despite targeting children, PCV7 vaccination had a beneficial and sustained effect on pneumonia-related hospitalisation rates, length of inpatient stay and mortality across all age groups. The greatest effects were seen at the extremes of age; in children younger than 2 years old (43.2\% (95\% CI 34.9 to 51.6) fewer admissions), and above 85 years of age (22.8\% (95\% CI 17.3 to 28.4$)$ ). An estimated 168000 fewer admissions for pneumonia were seen in 2009 , an effect attributed to this vaccine.

Reduction in pneumococcal carriage, and thereby transmission, is postulated to have resulted in the observed 168000 fewer admissions in 2009 attributed to the PCV7. A 13-valent pneumococcal vaccine is now available and has been introduced in the USA. It is unclear whether this will result in a further decrease in hospitalisations for pneumonia. ${ }^{1}$

- Griffin MR, Zhu Y, Moore MR, et al. U.S. Hospitalizations for pneumonia after a decade of pneumococcal vaccination. N Engl J Med 2013;369:155-63.

\section{W Jones}

Correspondence to Dr W Jones, CT2, University Hospital of Wales, Medical Directorate, Heath Park, Cardiff CF14 4XW, UK; jzm4wdj@doctors.org.uk

Competing interests None.

Provenance and peer review Not commissioned; internally peer reviewed.

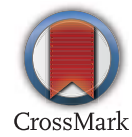

To cite Jones W. Thorax 2014;69:622. 